\title{
Acquired common variable hypogammaglobulinaemia and lymphoedema
}

\author{
S. Lozewicz, ${ }^{1}$ G. Morris, ${ }^{2}$ N. Garbett, ${ }^{1}$ N. Browse, ${ }^{2}$ B. Slavin ${ }^{3}$ and P. Cole ${ }^{1}$ \\ ${ }^{1}$ Department of Thoracic Medicine, Cardiothoracic Institute, Brompton Hospital, London SW3 $6 \mathrm{HP}$ and \\ ${ }^{2}$ Departments of Surgery and ${ }^{3}$ Clinical Chemistry, St. Thomas' Hospital, London SE1, UK.
}

\begin{abstract}
Summary: Two patients with lymphoedema of the legs developed recurrent respiratory infections and were found to have panhypogammaglobulinaemia. The early recognition and treatment of this form of immunity deficiency in patients with lymphoedema is important in order to prevent recurrent infections which could cause pulmonary damage and further lymphatic stasis.
\end{abstract}

\section{Introduction}

Acquired common variable hypogammaglobulinaemia, also known as varied immunodeficiency, ${ }^{1}$ is a condition of unknown cause which characteristically presents with recurrent sinusitis and pulmonary infections. A number of associated disorders have been described, including noncaseating granulomata, ${ }^{2,3}$ sarcoidosis, ${ }^{4}$ and autoimmune blood dyscrasias. ${ }^{5}$ We report two patients in whom acquired common variable hypogammaglobulinaemia was associated with primary lymphoedema of the lower limbs.

\section{Case reports}

Case 1

At the age of 26 this patient developed swelling of both legs which was more marked on the left. She underwent lymphography of the left leg at the age of 31 and this demonstrated hypoplasia of the proximal lymphatic vessels. Her legs became progressively more swollen and at the age of 39 she attended St. Thomas' Hospital for further investigation. Radioisotope lymphangiography with 99-technetium-labelled sulphur colloid in March 1984 demonstrated abnormal uptake in both legs compatible with a diagnosis of bilateral lymphoedema, and she was treated with Sigvaris elastic stockings. During the following year she had three episodes of pneumonia and was referred to the Brompton Hospital. She gave a history of

Correspondence: S. Lozewicz, M.D., M.R.C.P., St. Bartholomew's Hospital, London EC1A 7BE, UK. Accepted: 25 June 1987 anterior and posterior nasal discharge for as long as she could remember, and a cough productive of purulent sputum for approximately five years. There was no family history of immunity deficiency disorder or limb swelling.

On examination there was bilateral non-pitting oedema of the legs, and splenomegaly to $3 \mathrm{~cm}$ below the costal margin. There was no lymph node enlargement, hepatomegaly or stigmata of chronic liver disease. Investigations showed haemoglobin $12.8 \mathrm{~g} / \mathrm{dl}$, lymphocytes $0.4 / \mathrm{nl}$, neutrophils $2.1 / \mathrm{nl}$, platelets $137 / \mathrm{nl}$. Erythrocyte sedimentation rate $3 \mathrm{~mm} / \mathrm{h}$. Serum IgG $3.3 \mathrm{~g} / \mathrm{l}$ (normal 6.4-16), IgA $0.14 \mathrm{~g} / 1 \quad(0.7-3.2), \operatorname{IgM} 0.17 \mathrm{~g} / 1 \quad(0.56-3.52)$. Serum total bilirubin $19 \mu \mathrm{mol} / 1 \quad(2-15), \quad$ alkaline phosphatase 582 IU/1 (50-200), gamma-glutamyl transpeptidase $73 \mathrm{IU} / 1 \quad(3-14)$, aspartate transaminase 19 IU/1 (5-17), total protein $63 \mathrm{~g} / 1(62-82)$, albumin $38 \mathrm{~g} / 1 \quad(30-48)$. Prothrombin ratio 1.2 . Serum phosphate and corrected calcium were in the normal range. Chest X-ray showed bilateral abnormal shadowing and associated bronchial wall thickening in the mid and lower zones. Sinus Xrays demonstrated opacification of both maxillary antra.

In order to exclude other causes of both splenomegaly and the antibody deficiency the following tests were made and found to be negative: monospot for infectious mononucleosis, hepatitis B surface antigen, cytomegalovirus CFT, brucella and toxoplasma agglutination, and antinuclear antibodies. Bone marrow examination showed increased erythropoiesis and granulopoiesis and increased numbers of megakaryocytes; alcohol and acid-fast bacilli were not seen and culture for mycobacteria was negative.

(C) The Fellowship of Postgraduate Medicine, 1988 
Since intestinal lymphangiectasia can present with lymphoedema and hypogammaglobulinaemia, jejunal biopsy was performed but found to be normal. Liver biopsy showed non-necrotizing epithelioid and giant cell granulomata scattered throughout the hepatic parenchyma. A Kveim test was subsequently performed and found to be positive. Serum angiotensin converting enzyme activity was elevated to $80 \mathrm{nmol} / \mathrm{ml} / \mathrm{min}$ (normal 16-52) and a 1/100 dilution Mantoux test was negative.

In view of her frequent respiratory infections, the patient was started on replacement therapy with once weekly intramuscular injections of human gamma globulin in April 1985. Seven months after starting this treatment she had had only one further respiratory infection requiring treatment with antibiotics.

\section{Case 2}

This patient was well until approximately 15 years of age when she developed gradually progressive swelling of her left lower leg. In March 1983, at the age of 24, bipedal contrast lymphography demonstrated hypoplasia of the distal lymphatic vessels on the left and enlarged lumbar lymph nodes. The lymphatic vessels in the right leg were normal. A diagnosis was made of primary lymphoedema and she was treated with a Sigvaris elastic stocking. While undergoing investigation she acted as a control in a virology study as a result of which it was found that her serum immunoglobulin levels were low: IgG $1.0 \mathrm{~g} / 1, \operatorname{IgA}<0.2 \mathrm{~g} / 1, \operatorname{IgM}$ $<0.2 \mathrm{~g} / 1$.

She was referred to the Brompton Hospital in 1985 for further management of her hypogammaglobulinaemia. It was noted that she had had four episodes of cough productive of green sputum during the preceding 18 months. Between episodes she had an anterior and posterior nasal discharge in the summer months. There was no family history of limb swelling or immunity deficiency. On examination there was moderate non-pitting oedema of the left lower leg and the spleen was just palpable.

Investigations showed normal serum IgG $0.28 \mathrm{~g} / 1$, $\operatorname{IgA} 0.02 \mathrm{~g} / 1, \operatorname{IgM} 0.13 \mathrm{~g} / \mathrm{l}$. Serum total protein was $63 \mathrm{~g} / \mathrm{l}$, albumin $40 \mathrm{~g} / \mathrm{l}$. Chest X-ray was normal. Sinus X-rays demonstrated minimal mucosal thickening in the left maxillary antrum. Jejunal biopsy was normal and in particular demonstrated no evidence of intestinal lymphangiectasia.

The patient was started on replacement therapy with once weekly intramuscular injections of gamma globulin.

\section{Discussion}

Recurrent pulmonary infections and splenomegaly occurred in both patients and are characteristic features of acquired common variable hypogammaglobulinaemia. $^{6}$ Lymphography in patient 2 demonstrated lymph node enlargement, also a characteristic feature, ${ }^{6}$ and both patients demonstrated lymphopenia which does not occur in most cases but which has been reported previously. ${ }^{7}$ Hypersplenism has also been described $^{2}$ and may have caused the transient pancytopenia observed in patient 2 . Non-caseating granulomata occur in hypogammaglobulinaemia in sites including the lungs, liver, spleen and skin. ${ }^{6}$ There was histological evidence of hepatic granulomata in patient 1 and the computed tomographic scan of this patient was compatible with their presence in the lungs. This patient also had a positive Kveim test, elevated levels of serum angiotensin converting enzyme and a negative Mantoux test, thereby satisfying the definition of sarcoidosis. The association of sarcoidosis with hypogammaglobulinaemia has been reviewed by Lean et al., ${ }^{4}$ but in general, immunoglobulin levels are elevated in patients with sarcoidosis. ${ }^{8}$ In this context, recent work $^{9}$ suggests that granuloma formation in sarcoidosis, as exemplified by the Kveim reaction, could be a host response to compensate for impaired delayed hypersensitivity which can often be demonstrated in acquired common variable hypogammaglobulinaemia.

Damage to lymphatic vessels and subsequent lymphoedema can result from local infection such as cellulitis ${ }^{10}$ which is common in patients with hypogammaglobulinaemia. ${ }^{11}$ It is unlikely that the lymphoedema in our patients followed infective damage to lymphatic vessels since there was no history of local infection preceding the development of limb swelling in either case, and the lymphographic appearances were those of primary lymphoedema. Kinmonth and co-workers ${ }^{10}$ divide this condition into three types on the basis of the patient's age at onset: congenital lymphoedema has its onset at birth, lymphoedema praecox before the age of 35 years, and lymphoedema tarda after 35 . Both patients reported here had lymphoedema praecox.

Intestinal lymphangiectasia may present with lymphoedema and hypogammaglobulinaemia but the normal jejunal biopsy and normal serum albumen in each patient make this diagnosis unlikely. Lymphoedema also occurs in association with recurrent pleural effusions and yellow dystrophic nails in the 'yellow nail syndrome'12,13 One of this triad of features may occur in isolation, 
many years before the appearance of others. ${ }^{14}$ Although neither of our patients had nail changes or pleural effusions, it is not possible to exclude the possibility that they are early examples of the 'yellow nail syndrome'. Immunodeficiency is not a characteristic feature of this syndrome but Siegelman et al. ${ }^{15}$ reported a case with low levels of serum gamma globulin in whom Ig classes were not measured, and a further case with absence of IgA on immunoelectrophoresis has been described. ${ }^{16}$

Both our patients had sinusitis and there were features of bronchiectasis in patient 1 . Both these conditions occur in association with hypogammaglobulinaemia ${ }^{6}$ and also in association with primary lymphoedema in patients with the yellow nail

\section{References}

1. World Health Organization Technical Reports Series 630. Immunodeficiency. Geneva, 1978.

2. Prasad, A.S., Reiner, E. \& Watson, C.J. Syndrome of hypogammaglobulinaemia, splenomegaly and hypersplensim. Blood 1957, 12: 926-932.

3. Perks, W.H. \& Peterham, I.S. Familial combined cellular and humoral immune defect with multisystem granulomata. Thorax 1978, 33: 101-105.

4. Leen, C.L.S., Bath, J.C.J.L., Brettle, R.P. \& Yap, P.L. Sarcoidosis and hypogammaglobulinaemia: a report of two cases and a review of the literature. Sarcoidosis 1985, 2: 91-95.

5. Webster, A.D., Platts-Mills, T.A., Jannossy, G., Morgan, M. \& Asherson, G.L. Autoimmune blood dyscrasias in five patients with hypogammaglobulinaemia: Response of neutropenia to vincristine. $J$ Clin Immunol 1981, 1: 113-118.

6. Rosen, F.S. \& Janeway, C.A. The gammaglobulins: The antibody deficiency syndromes. $N$ Engl $J$ Med 1966, 275: 709-715.

7. Gajl-Peczalska, K.J., Park, B.H., Biggar, W.D. \& Good, R.A. B and $T$ lymphocytes in primary immunodeficiency disease in man. J Clin Invest 1973, 52: 919-928.

8. Scadding, J.G. Immunology of sarcoidosis. In: Gell, P.G., Coombes, R.R. \& Lachman, P.J. (eds) Clinical Aspects of Immunology. Blackwell Scientific, London, 1975, pp 1061-1077.

9. Munro, C.S., Mitchell, D.N., Poulter, L.W. \& Cole, P.J. Immunological processes active in developing Kveim responses differ in normal and sarcoidosis subjects. Am Rev Resp Dis 1986, 133: A244 (abstract). syndrome and normal immunoglobulin levels. ${ }^{16,17}$

The cases reported here provide a further example of acquired common variable hypogammaglobulinaemia with features satisfying the definition of sarcoidosis, ${ }^{4}$ and demonstrate an association between this form of immunity deficiency and lymphoedema praecox. Cellulitis is more common in patients with lymphoedema and may cause damage to lymphatic vessels and progression of lymphatic stasis. ${ }^{10}$ The early recognition and treatment of hypogammaglobulinaemia in patients with lymphoedema may be important in preventing this cycle of damage, as well as being crucial to the prevention of lung damage due to recurrent or chronic infection.

10. Kinmonth, J.B. The Lymphatics: Surgery, Lymphography and Diseases of the Chyle and Lymph Systems. Edward Arnold, London, 1982. p 84.

11. Medical Research Council Working Party on Hypogammaglobulinaemia. Hypogammaglobulinaemia in the United Kingdom. Special report series 310, HMSO, London, 1971, p 12.

12. Sammon, P.D. \& White, W.F. The 'yellow nail syndrome'. Br J Dermatol 1964, 76: 153-157.

13. Emerson, P.A. Yellow nails, lymphoedema and pleural effusions. Thorax 1966, 21: 247-253.

14. Eastwood, H.D. \& Williams, T.J. Pleural effusions and yellow nails of late onset. Postgrad Med J 1973, 49: 364-365.

15. Siegelman, S.S., Heckman, B.H. \& Hasson, J. Lymphoedema, pleural effusions and yellow nails. Associated immunologic deficiency. Dis Chest 1969, 56: 114-117.

16. Nakielna, E.M., Wilson, J. \& Ballon, H.S. Yellow nail syndrome: Report of three cases. Can Med Assoc J 1976, 115: 46-48.

17. Hiller, E., Rosenow, E. \& Olsen, A. Pulmonary manifestations of the yellow nail syndrome. Chest 1972, 61: 452-458. 